\title{
Quantum-Dynamical Theory of Electron Exchange Correlation
}

\author{
Burke Ritchie $^{1,2}$ and Charles A. Weatherford ${ }^{3}$ \\ ${ }^{1}$ Lawrence Livermore National Laboratory, Livermore, CA 94550, USA \\ ${ }^{2}$ Livermore Software Technology Corporation, Livermore, CA 94550, USA \\ ${ }^{3}$ Department of Physics, Florida A\&M University, Tallahassee, FL 32307, USA \\ Correspondence should be addressed to Burke Ritchie; ritchie@lstc.com
}

Received 4 November 2012; Revised 13 January 2013; Accepted 14 January 2013

Academic Editor: Benjaram M. Reddy

Copyright (C) 2013 B. Ritchie and C. A. Weatherford. This is an open access article distributed under the Creative Commons Attribution License, which permits unrestricted use, distribution, and reproduction in any medium, provided the original work is properly cited.

\begin{abstract}
The relationship between the spin of an individual electron and Fermi-Dirac statistics (FDS), which is obeyed by electrons in the aggregate, is elucidated. The relationship depends on the use of spin-dependent quantum trajectories (SDQT) to evaluate Coulomb's law between any two electrons as an instantaneous interaction in space and time rather than as a quantum-mean interaction in the form of screening and exchange potentials. Hence FDS depends in an $a b$ initio sense on the inference of SDQT from Dirac's equation, which provides for relativistic Lorentz invariance and a permanent magnetic moment (or spin) in the electron's equation of motion. Schroedinger's time-dependent equation can be used to evaluate the SDQT in the nonrelativistic regime of electron velocity. Remarkably FDS is a relativistic property of an ensemble of electron, even though it is of order $c^{0}$ in the nonrelativistic limit, in agreement with experimental observation. Finally it is shown that covalent versus separated-atoms limits can be characterized by the SDQT. As an example of the use of SDQT in a canonical structure problem, the energies of the ${ }^{1} \Sigma_{g}$ and ${ }^{3} \Sigma_{u}$ states of $\mathrm{H}_{2}$ are calculated and compared with the accurate variational energies of Kolos and Wolniewitz.
\end{abstract}

\section{Introduction}

One may consider that quantum chemistry is dominated by theoretical and computational efforts to achieve an accurate description of electron exchange correlation, evolving such workhorse methodologies as Hartree-Fock-Configuration Interaction, Density Functional Theory, and numerous variations on the theme of nonrelativistic quantum mechanics applied to problems of chemical interest. But yet, owing to historical happenstance, more heat than light has been generated concerning the fundamental physical understanding of exchange-correlation. Even in early calculations in which correlation was built into the wave function it was recognized that the concept of exchange tended to lose meaning in a calculation in which correlation was treated to high accuracy [1]. As another example it was shown that a high-order perturbation calculation in which the electronelectron interaction is treated as the perturbation is able to achieve order by order the correct permutation symmetry of the wave function starting in zeroth order with a simple unsymmetrized product of orbitals [2]. This conundrum can be easily understood for the two-electron, one-nucleus problem by a simple change from nucleus centered to Jacobi coordinates in which one vector connects the two electrons, and the other connects the center of mass of the two electrons to the nucleus (where of course it is understood that the Born-Oppenheimer picture is being used). Then the wave function separates naturally into two linearly-independent wave functions which are either even or odd in the exchange of electrons. In other words the new choice of coordinates in which correlation is achieved through a physically judicious choice of coordinates also achieves the correct exchange symmetry, which is guaranteed by the symmetry of the Hamiltonian with respect to the electron exchange.

Nonrelativistic quantum theory fails us, however, even for two-electron problems. According to experimental observation, the electrons have intrinsic angular momentum comprising two-spin-1/2 states, such that the total wave function must be antisymmetric on electron exchange, which is satisfied either by a product of the even spatial wave function adduced above with a spin state which is odd on electron exchange (singlet state) or by a product of the 
adduced odd spatial wave function with a spin state which is even on electron exchange (triplet state). The generalization is of course the Slater determinantal wave function of spin orbitals which guarantees the correct antisymmetric permutation symmetry for $N$ spin-1/2 particles. Hence the standard methodologies [3], for all their success, simulate many-electron quantum states in an ad hoc manner, based on experimental observation, but tell us nothing fundamental about the physical basis of how the spin state of an individual electron is related to the Pauli exclusion principle and the observed Fermi-Dirac statistical behavior of an aggregate of electrons.

\section{Spin-Dependent Quantum Trajectories and Electron Exchange Correlation}

Readers should recognize that an electron's spin state and its spatial correlation with another electron are strongly related, as suggested by our observation that a successful simulation of correlation is also accompanied by the correct exchange symmetry. Notice, however, that such a relationship between electron spin and the electron-electron Coulomb interaction appears to be grossly at odds with our intuitive understanding of quantum chemistry, likely due to the absence of a particletrajectory picture in the standard methodologies. Bohm formulated a quantum dynamical approach in which the phaseamplitude solution of Schroedinger's equation has a formal relationship to classical hydrodynamics $[4,5]$. Bohmian dynamics has recently undergone a renewed interest [6]. It is a method of solving the time-dependent Schrodinger equation by assuming a form for the wavefunction $\Psi(x, t)=$ $R(x, t) e^{i S(x, t)}$ where $R$ (such that $R$ is greater than or equal to zero for all $x$ ) is the amplitude and $S$ is the action function. Then upon substitution into the time-dependent Schroedinger equation, two coupled equations (continuity equation and the quantum Hamilton-Jacobi equation) are obtained. The net result is to produce quantum trajectories. These equations have proven to be difficult to solve accurately, particularly for multi electrons [6]. In fact, it is difficult to solve in three spatial dimensions for one electron. Note that in the context of the Bohmian equations, the discretization is done in terms of what are called pseudo particles. Thus, when it is said that say fifty particles are propagated, it typically means fifty pseudo particles are used to describe the dynamics of one real particle. As far as we are aware, no one has computed the dynamics of two real particles using Bohmian dynamics, and in fact, it does not seem possible to do so with current algorithms and computer hardware.

We therefore seek a quantum trajectory approach for real electrons in which the spin of an individual electron is correctly accounted for, which means that we must look to Dirac's rather than to Schroedinger's equation. One of the desiderata of Dirac's program [7] for incorporating Einstein's special theory of relativity into quantum mechanics was that the quantum analog of the classical equation of continuity,

$$
\frac{\partial \rho}{\partial t}+\vec{\nabla} \cdot \vec{j}=0
$$

should be inferred from the equation of motion for the relativistic electron,

$$
\begin{aligned}
& \left(i \hbar \frac{\partial}{\partial t}-V-m c^{2}\right) \psi(\vec{r}, t)+i \hbar c \vec{\sigma} \cdot \vec{\nabla} \chi(\vec{r}, t)=0, \\
& \left(i \hbar \frac{\partial}{\partial t}-V+m c^{2}\right) \chi(\vec{r}, t)+i \hbar c \vec{\sigma} \cdot \vec{\nabla} \psi(\vec{r}, t)=0,
\end{aligned}
$$

whereupon [7],

$$
\vec{j}(\vec{r}, t)=c\left[\psi^{+}(\vec{r}, t) \vec{\sigma} \chi(\vec{r}, t)+\chi^{+}(\vec{r}, t) \vec{\sigma} \psi(\vec{r}, t)\right],
$$

where $\vec{\sigma}$ is Pauli's vector, and the quantum density is

$$
\rho(\vec{r}, t)=\psi^{+}(\vec{r}, t) \psi(\vec{r}, t)+\chi^{+}(\vec{r}, t) \chi(\vec{r}, t),
$$

where $\psi(\vec{r}, t)$ and $\chi(\vec{r}, t)$ are the large and small Dirac spinors, respectively, and the superscripts denote Hermitian conjugates. On eliminating (2b) in favor of (2a) and dropping all terms of order $c^{-2}$, which is the nonrelativistic limit, Schroedinger's equation is recovered for $\psi$, which was another desideratum in Dirac's program for a relativisticelectron theory. Now notice that a velocity field can be inferred from the current by writing

$$
\begin{aligned}
\vec{j}(\vec{r}, t) & =c\left[\psi^{+}(\vec{r}, t) \vec{\sigma} \chi(\vec{r}, t)+\chi^{+}(\vec{r}, t) \vec{\sigma} \psi(\vec{r}, t)\right] \\
& =\vec{v}(\vec{r}, t) \rho(\vec{r}, t),
\end{aligned}
$$

and solving for $\vec{v}(\vec{r}, t)$, from which a trajectory, $\vec{s}(t)$, can be calculated from the time integration of the velocity field to find a position field,

$$
\vec{q}(\vec{r}, t)=\int_{0}^{t} d t^{\prime} \vec{v}\left(\vec{r}, t^{\prime}\right),
$$

and finally by finding the quantum expectation value of the position field,

$$
\begin{aligned}
\vec{s}(t)=\int d \vec{r}\left[\psi^{+}(\vec{r}, t) \vec{q}(\vec{r}, t) \psi(\vec{r}, t)\right. \\
\left.+\chi^{+}(\vec{r}, t) \vec{q}(\vec{r}, t) \chi(\vec{r}, t)\right] .
\end{aligned}
$$

One more step is needed to show the relationship of electron spin and electron exchange correlation, namely, the evaluation of the electron-electron Coulomb interaction using quantum trajectories. Considering the case of two electrons, one of which is described by (1)-(7). The Coulomb interaction for an electron with position vector $\vec{r}$ with the other electron with position vector $\vec{r}^{\prime}$ can then be written as follows:

$$
V_{e e^{\prime}}(\vec{r}, t)=\frac{e^{2}}{\left|\vec{r}-\vec{s}^{\prime}(t)\right|}
$$

Similarly the electron at $\vec{r}^{\prime}$ is described by (1)-(7) in which $\vec{r}$ is replaced by $\vec{r}^{\prime}$ and the interaction is given by

$$
V_{e^{\prime} e}\left(\vec{r}^{\prime}, t\right)=\frac{e^{2}}{\left|\vec{r}^{\prime}-\vec{s}(t)\right|} \text {. }
$$




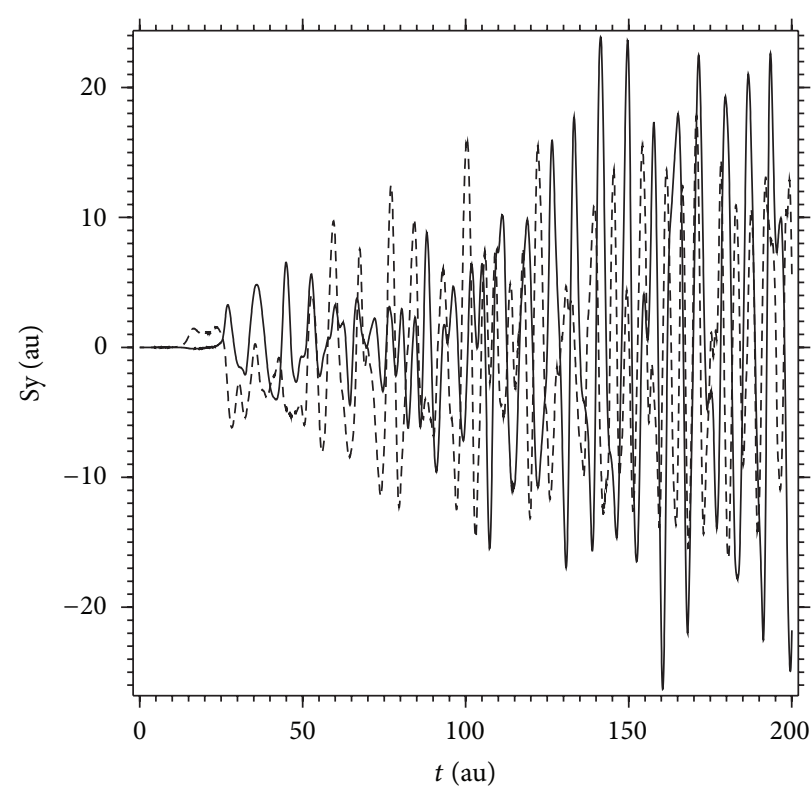

FIgURE 1: Quantum trajectories in the y direction at $R=1.4 \mathrm{au}$ showing the correlation of the two electrons in the ${ }^{1} \Sigma_{g}$ state of $\mathrm{H}_{2}$. Solid: spin-up electron. Dotted: spin-down electron. The trajectories are calculated from (7) in the nonrelativistic limit using spectral wave functions.

Thus the electron-electron potential is evaluated as an instantaneous interaction in space and time rather than as a quantum-mean interaction in the form of Coulomb and exchange integrals. This is a critical step. Normally the interaction is written as $v_{e e^{\prime}}=e^{2} /\left|\vec{r}-\vec{r}^{\prime}\right|$, which is a function in 6-space well known to be incompatible with Lorentzinvariance [8], which is a requirement for a correct relativistic theory. The authors' comment [8], following their equation (38.4), "the Coulomb term [e-e' interaction] in (38.3), however, is not even approximately Lorentz invariant, and relativistic corrections to the interaction between the two electrons are furnished by quantum electrodynamics," refers to the Lorentz-invariant form of the e-e $e^{\prime}$ interaction given by its representation in QED in terms of the exchange of virtual photons. Notice that in the present paper we are claiming that these relativistic corrections, at least for nonradiative interactions, are given by representing the $\mathrm{e}-\mathrm{e}^{\prime}$ or $\mathrm{e}^{\prime}-\mathrm{e}$ interactions in the forms given by (8)-(9) and by calculating the trajectories using (2a)-(7). This is so because the 4-space form of (8)(9) is fully compatible with the Lorentz invariance of Dirac's one-electron equation as given by (2a) and (2b), in which $V$ is explicitly time dependent and can be written as $V(\vec{r}, t)$. In other words the time-independent 6 -space potential, $v_{e e^{\prime}}=$ $e^{2} /\left|\vec{r}-\vec{r}^{\prime}\right|$, is represented in 3 -space and the time by using the position vector of one electron and the trajectory of the other. Readers will note that Dirac's one-electron equation, as given for example in the literature for the hydrogen atom, is exact and therefore Lorentz invariant for the Coulomb attraction to the nucleus, $V(r)=-Z e^{2} / r$. It is still exact and Lorentz invariant for the general scalar potential $V(\vec{r}, t)$. This is a true statement because in the electron's 4-momentum, $(\gamma m c, \gamma m \vec{v})=(\gamma m c, \vec{p})=(\gamma m c,-i \hbar \vec{\nabla})$, the scalar part can be expressed as terms of $(1 / c)[i \hbar(\partial / \partial t)-V(\vec{r}, t)]$ on using the operator form of the classical relativistic Hamiltonian $i \hbar(\partial / \partial t)=\gamma m c^{2}+V(\vec{r}, t)$, where $\gamma$ is the Lorentz factor $\gamma=\sqrt{1+\left(p^{2} / m^{2} c^{2}\right)}$. We can only guess that workers have not used the present methodology to find a fully relativistic, Lorentz-invariant theory for many fermions due historically to the dominance of time-independent quantum theory in absence of an explicitly time-dependent interaction such as the vector potential, $\vec{A}(\vec{r}, t)$, in matter-radiation physics, in which case the 4-momentum is written $(\gamma m c,-i \hbar \vec{\nabla}$ $(e / c) \vec{A}(\vec{r}, t))$. Equations (8)-(9) are functions of 3-space and the time and are fully compatible with the Lorentz invariance of Dirac's one-electron Hamiltonian and with the Lorentz covariance of his one-electron wave equation [7]. Notice that we do not venture onto the ice of two-body Dirac theory [9]. Proceeding heuristically we believe that it is possible to derive a correct many-electron relativistic quantum theory simply by replacing Newton's equation of motion for each electron in an aggregate of electrons by Dirac's equation of each electron in the aggregate, thusly for an electron whose position vector is $\vec{r}$ interacting with another electron whose position vector is $\vec{r}^{\prime}$,

$$
\begin{aligned}
\frac{d \vec{p}(t)}{d t}=-\vec{\nabla} \frac{e^{2}}{\left|\vec{r}(t)-\vec{r}^{\prime}(t)\right|} \longrightarrow & \\
i \hbar \frac{\partial \psi_{D}(\vec{r}, t)}{\partial t}= & {\left[-i \hbar c \vec{\alpha} \cdot \vec{\nabla}+\beta m c^{2}\right.} \\
& \left.+\frac{e^{2}}{\left|\vec{r}-\vec{s}^{\prime}(t)\right|}\right] \psi_{D}(\vec{r}, t),
\end{aligned}
$$

where $\vec{p}=\gamma m(d \vec{r}(t) / d t), \gamma=\sqrt{1+\left(p^{2} / m^{2} c^{2}\right)}, \psi_{D}=\left(\begin{array}{c}\psi \\ \chi\end{array}\right)$, $\vec{\alpha}=\left(\begin{array}{cc}0 & \vec{\sigma} \\ \vec{\sigma} & 0\end{array}\right), \beta=\left(\begin{array}{cc}I & 0 \\ 0 & -I\end{array}\right)$, and $I$ is the $2 \times 2$ identity matrix. Equations (2a) and ( $2 b$ ) are identical to the right-hand side of the arrow in (10), which is just another way of writing Dirac's equation.

Pauli's exclusion principle is obeyed if (2a) and (2b) and their counterparts for a second electron are evolved in the time for identical spatial functions and opposite spin states at initial time (Figure 1), which corresponds to a singlet state or for different spatial functions and the same spin states at initial time (Figure 2), which corresponds to a triplet state. Notice that Figure 1 shows quantum trajectories with slightly different frequencies, which can happen because spatial wave functions which are identical at initial time can develop differences over time, in analogy to the use of unrestricted Hartree-Fock theory in standard time-independent theory. It should be noted that, although one must choose a set of initial conditions for the time integration of the equations, the problem is not an initial-value problem in the same sense as in classical mechanics in which, due to the deterministic nature of the trajectories, one must find the physical result as an average over a set of different initial conditions since we cannot know a unique set of initial conditions for the particles. The reason can be found in the Heisenberg uncertainty principle, $\Delta E \Delta t \geq \hbar$, which governs the time 
integration of the quantum wave equations: near initial time the energy is very broad and narrows with increasing time to give a spectrum of energy levels (Figure 3). Quantum dynamics, however, does share with classical dynamics the property that stationary (i.e., constant energy) solutions are obtained.

The envelop of trajectory amplitudes shown in Figure 1 is observed to increase over time because the quantum trajectories (hereafter called spectral trajectories) are calculated using the time-dependent solutions (hereafter called the spectral solutions [10]), which are a superposition of quantum states including the continuum, such that the envelop growth over time reflects the excited and continuum content of the wave function. On the other hand the envelop of trajectory amplitudes shown in Figure 4 is not observed to increase over time because the trajectories (hereafter called eigentrajectories) are calculated using eigenfunctions as filtered from the spectral solutions at a specific stationary energy as determined from Figure 3, as described in [10]. In some of the calculations Hamiltonian expectation values calculated using eigenfunctions and eigentrajectories (Figure 5) still exhibit an oscillation in the time. In these cases the physical energies can be found from the time average of the Hamiltonian expectation values (Figures 5 and 6); in other words the time average is sensibly stationary.

\section{Quantum Trajectories in the Nonrelativistic Limit}

The time-independent solution of (2b) can be written as

$$
\chi(\vec{r}, t)=\frac{-i \hbar c \vec{\sigma} \cdot \vec{\nabla} \psi(\vec{r}, t)}{E-V+m c^{2}} .
$$

Evaluating $\chi(\vec{r}, t)$ in the nonrelativistic limit, for which $(E-$ $V) \ll m c^{2}$, we immediately see that the current given by (3) is of order $c^{0}$ in this limit. Remarkably the $c^{0}$ order of the quantum trajectories is in agreement with experimental observation that Fermi-Dirac statistics is obeyed for all electron velocities, including those whose Dirac current is calculated in the regime of nonrelativistic velocity.

The trajectories are quantum mechanical since they can be calculated either using Dirac's equation in the regime of relativistic velocities or Schrodinger's equation in the regime of nonrelativistic velocities. The quantum trajectories are spin dependent since they depend explicitly on Pauli's vector. Our result, for the first time, gives a relativistic correction which is of order $c^{0}$, all other relativistic corrections being of order $c^{-2}$. This relativistic correction is due exclusively to the spin of the electron and accounts for Fermi-Dirac statistics. Traditionally relativistic corrections have been considered to start at order $c^{-2}$ for atomic fine structure. Readers will appreciate the apparent paradox that we had to appeal to Dirac's equation to explain the omnipresence of electron spin in all fermionic phenomena notwithstanding the relativistic or nonrelativistic nature of the motion.

We believe that this unforeseen Dirac correction of order $c^{0}$, which is of course the same order as all Schroedinger's contributions to the Hamiltonian, is due to the Lorentz

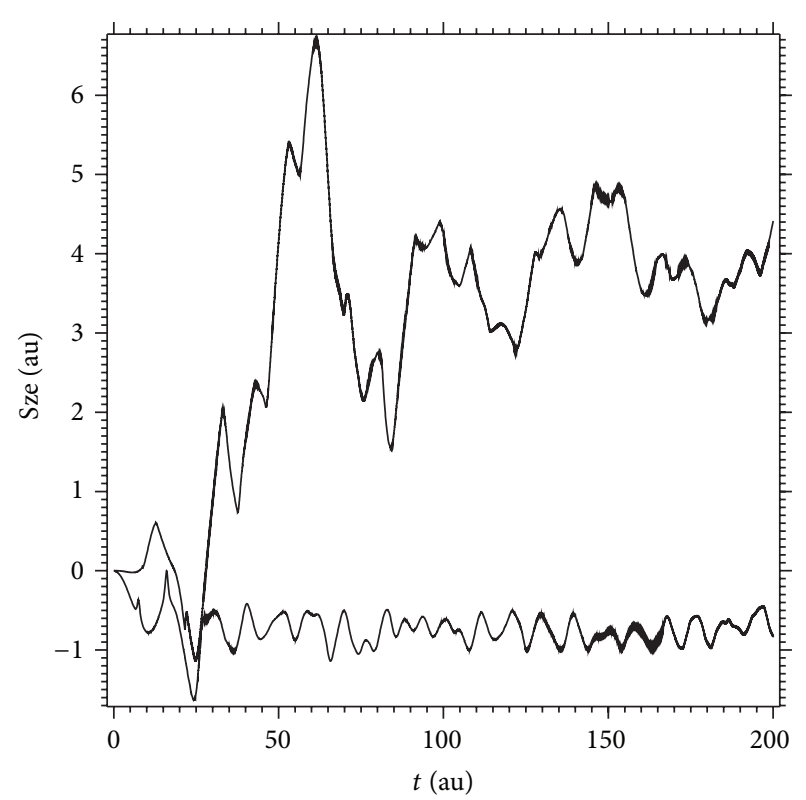

FIGURE 2: Quantum eigentrajectories in the $z$ direction showing how the two electrons of $\mathrm{H}_{2}$ correlate with increasing time in the formation of an antibonding state. Upper: spin-up electron. Lower: spin-up electron. The eigentrajectories are calculated from (7) in the nonrelativistic limit using eigenfunctions for two parallel spin states of the ${ }^{3} \Sigma_{u}$ state of $\mathrm{H}_{2}$.

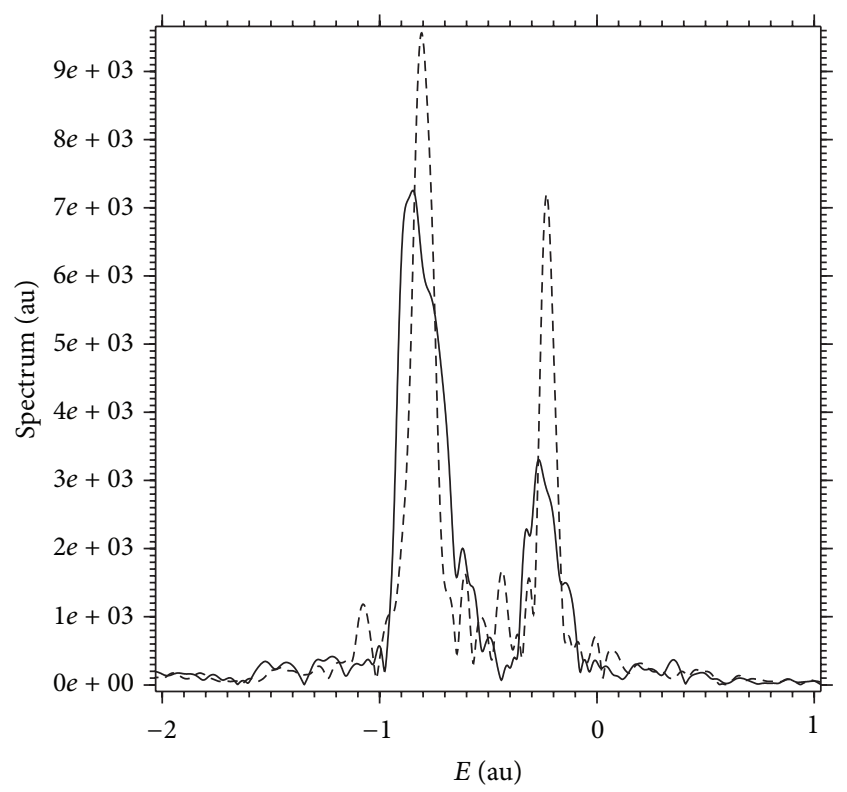

Figure 3: Spectrum at $R=2.5$ au for the ${ }^{1} \Sigma_{g}$ state of $\mathrm{H}_{2}$. Solid: spinup electron. Dotted: spin-down electron.

covariance [7] of Dirac's equation appropriate for a spin$1 / 2$ particle, as discussed in the last section. This surmise is supported by the fact that the Klein-Gordon relativistic equation appropriate for a spin- 0 particle, although it is Lorentz invariant, it fails to satisfy the equation of continuity given by (1) for a physically acceptable current. 


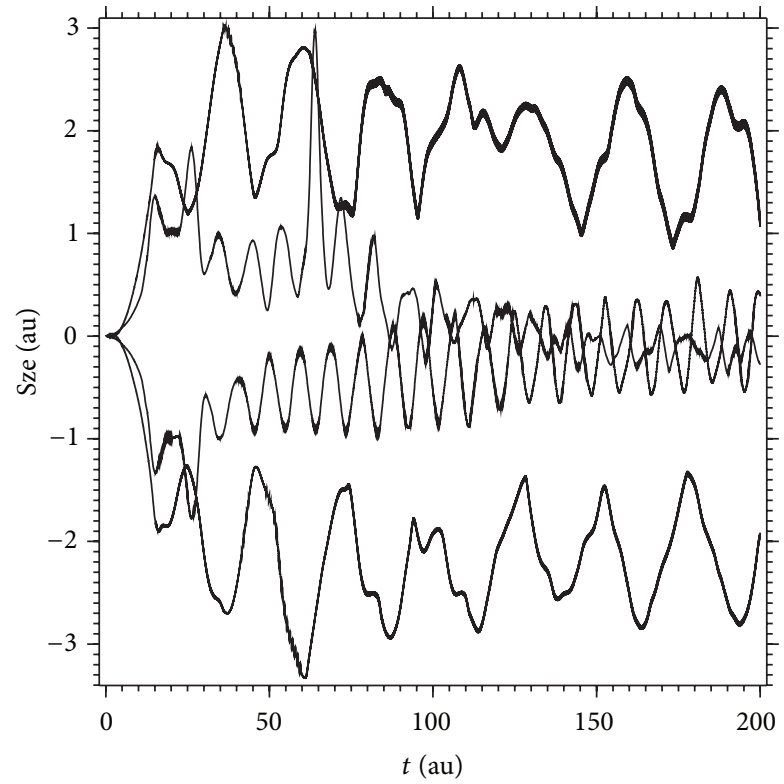

FIgURE 4: Quantum eigentrajectories in the $z$ direction. Inner curves: $R=1.4 \mathrm{au}$. Outer curves: $R=3.0 \mathrm{au}$. The inner curves show how the two electrons correlate with increasing time and eventually find the region of covalent bonding located between the two protons fixed at $z= \pm 0.7 \mathrm{au}$, while the outer curves show that the electrons remain in the vicinity of the separated atoms for all times. The eigentrajectories are calculated from (7) in the nonrelativistic limit using eigenfunctions for the up and down spin states for the ${ }^{1} \Sigma_{g}$ state of $\mathrm{H}_{2}$.

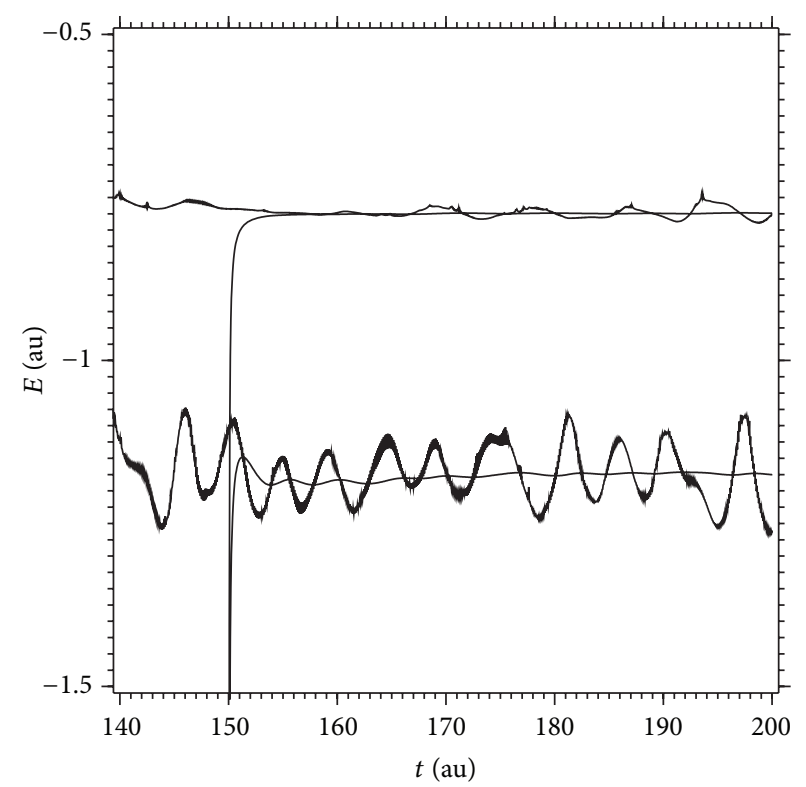

FIgURE 5: Expectation values of the Hamiltonian and their time averages for $R=1.4$ au toward the end of the time integration. Lower: ${ }^{1} \Sigma_{g}$ state. Upper: ${ }^{3} \Sigma_{u}$ state. These energies, especially for the ${ }^{1} \Sigma_{g}$ state, have some numerical noise (high-frequency hair superimposed on the wave oscillation), but nevertheless the time averages at maximum time agree well with the time-independent results, which are -1.174 and -0.7842 for the ${ }^{1} \Sigma_{g}$ and ${ }^{3} \Sigma_{u}$ states, respectively.

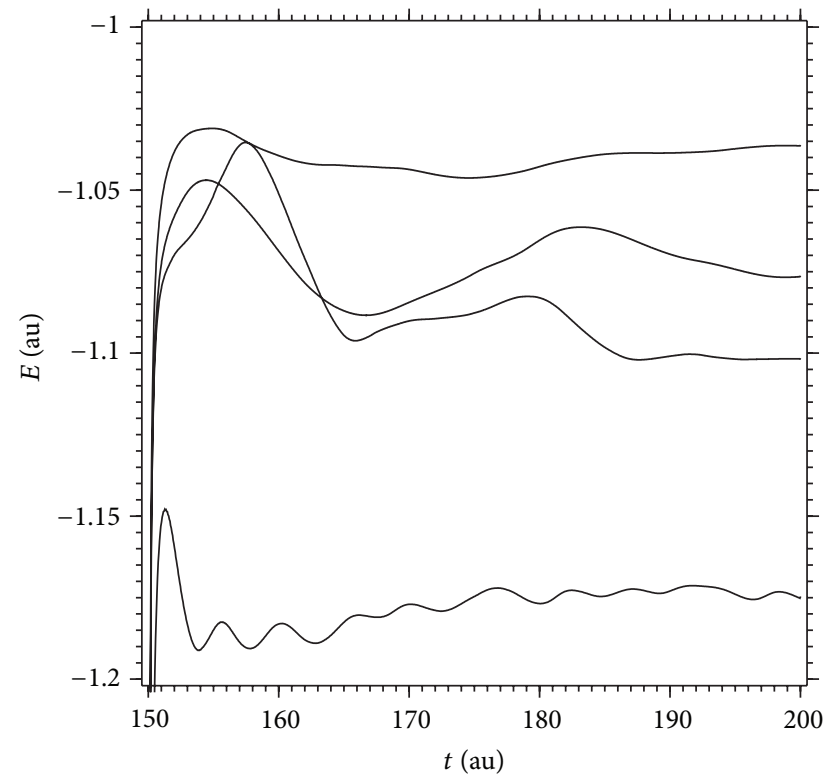

Figure 6: Time average of the Hamiltonian expectation values for the ${ }^{1} \Sigma_{g}$ state. From bottom to top for $R=1.4 \mathrm{au}, R=2.5 \mathrm{au}$, $R=3.0 \mathrm{au}$, and $R=4.0 \mathrm{au}$, respectively. The KW energies are, respectively, $-1.174 \mathrm{au},-1.094 \mathrm{au},-1.057 \mathrm{au}$, and $-1.016 \mathrm{au}$. The agreement with $\mathrm{KW}$ at the larger internuclear distances is not as good owing to smaller binding energies whose magnitude falls into the last digit of accuracy of the calculation.

In the nonrelativistic regime of electron velocity, (2a)(9) may be evaluated using Schroedinger's equation, which follows on exactly eliminating (2b) in favor of (2a) and dropping contributions in the resulting equation for the large component, $\psi(\vec{r}, t)$, of order $c^{-2}$. The current is evaluated in the nonrelativistic limit using $\psi(\vec{r}, t)=\psi_{S}(\vec{r}, t) \chi_{m_{s}}$ and $\chi(\vec{r}, t)$ given by (11) in the regime $(E-V) \ll m c^{2}$, where $\psi_{S}(\vec{r}, t)$ obeys the time-dependent Schroedinger's equation as follows:

$$
i \hbar \frac{\partial \psi_{S}(\vec{r}, t)}{\partial t}=\left[-\frac{\hbar^{2}}{2 m} \nabla^{2}+V(\vec{r}, t)\right] \psi_{S}(\vec{r}, t)
$$

$\chi_{m_{s}}$ has up (plus sign) or down (minus sign) magnetic substates denoted by $m_{s}= \pm 1 / 2$ (i.e., $\alpha$ or $\beta$ spin states), respectively. Written out explicitly in terms of the large component the current given by (3) becomes

$$
\begin{aligned}
\vec{j}(\vec{r}, t) \cong \frac{\hbar}{2 m}[ & -i\left(\psi_{S}^{*} \vec{\nabla} \psi_{S}-\psi_{S} \vec{\nabla} \psi_{S}^{*}\right) \\
& +\psi_{S}^{*} \chi_{m_{s}}^{+}(\vec{\nabla} \times \vec{\sigma}) \psi_{S} \chi_{m_{s}} \\
& \left.-\psi_{S} \chi_{m_{s}}^{+}(\vec{\sigma} \times \vec{\nabla}) \psi_{S}^{*} \chi_{m_{s}}\right]
\end{aligned}
$$


where we have used $\vec{\sigma}^{+}=\vec{\sigma}$ and the identity, $(\vec{\sigma} \cdot \vec{A})(\vec{\sigma} \cdot \vec{B})=$ $\vec{A} \cdot \vec{B}+i \vec{\sigma} \cdot(\vec{A} \times \vec{B})$, from which the identities useful in evaluating the current can be inferred as follows:

$$
\begin{aligned}
& \vec{\sigma}(\vec{\sigma} \cdot \vec{\nabla})=\vec{\nabla}+i(\vec{\nabla} \times \vec{\sigma}), \\
& (\vec{\sigma} \cdot \vec{\nabla}) \vec{\sigma}=\vec{\nabla}+i(\vec{\sigma} \times \vec{\nabla}) .
\end{aligned}
$$

Written out explicitly for up (upper sign) or down (lower sign) spin states the nonrelativistic current is

$$
\begin{aligned}
& \vec{j}_{n r}(\vec{r}, t)=\frac{\hbar}{m}[ \operatorname{Im} \psi_{S}^{*}(\vec{r}, t) \vec{\nabla} \psi_{S}(\vec{r}, t) \\
& \pm \hat{i} \operatorname{Re} \psi_{S}^{*}(\vec{r}, t) \frac{\partial}{\partial y} \psi_{S}(\vec{r}, t) \\
&\left.\mp \hat{j} \operatorname{Re} \psi_{S}^{*}(\vec{r}, t) \frac{\partial}{\partial x} \psi_{S}(\vec{r}, t)\right] .
\end{aligned}
$$

The first term on the right side of (15), which is independent of spin, is the familiar term contributed by Schroedinger's equation, while the second and third terms, which are transverse to the axis of quantization along $z$, are contributed uniquely by Dirac theory. Notice that all contributions to the current are of order $c^{0}$, as discussed earlier in this section.

\section{Calculations and Results}

The time-dependent Schroedinger's equation [(12)] is solved in the time and three Cartesian coordinates for each electron of $\mathrm{H}_{2}$ using an algorithm described previously [11]. (Preliminary He-atom calculations were presented previously [12].) The computational grid box is square with a uniform mesh of $32^{3}$. The accuracy of the calculation could be improved by mesh refinement of the uniform mesh or by use of an adaptive mesh (which we do not have at our disposal); however, we find that for $Z=1$ or $Z=2$ the present mesh is adequate, especially for the proof of principle calculations presented here. The two Schroedinger's equations were integrated in the time for a length of 200 au using 4000 mesh points (for internuclear distance $R=1.4$ au calculations) or 8000 mesh points (for all other internuclear distance calculations). At initial time a Slater-type orbital with form $r e^{-\zeta r}, \zeta=1 / 2$ centered on each proton is used for each electron for the ${ }^{1} \Sigma_{g}$ state and with forms $r e^{-\zeta r} \zeta=1$ and $r^{2} e^{-\zeta r}, \zeta=1 / 2$ for the electrons of the ${ }^{3} \Sigma_{u}$ state. The Pauli principle is enforced at initial time for electrons in the same spin state, and the electrons obey the Pauli principle for all subsequent times due to their mutual correlation. If the Pauli principle is violated at initial time by assigning the same spatial orbital to electrons in the same spin state, then the electrons fail to correlate for subsequent times.

The time-dependent wave function for each electron is evolved, and its spectrum of eigenvalues and eigenfunctions is obtained using the methods described in the pioneering paper of Feit et al. [10] on the spectral solution of the timedependent Schroedinger equation. The results are sensitive to initial conditions only in the sense that a poor choice (use of $r^{0}$ and larger values of $\zeta$, e.g.) was found to lead to trajectory run away and an unphysical solution. As in classical dynamics care should be taken to use energy-conserving numerical methods to integrate the trajectory fields using (6). Trajectory run away is easily recognized from a plot of trajectory versus time in which the trajectory is not periodic and grows with time, representing an unbound electron (self ionization) or in which it is periodic at large distances from the nuclei, representing a spurious excited state (self excitation). The energies of runaway trajectories are observed to lie below the true energies due to insufficient strength of the interelectronic potential. When sufficient care is taken to obtain energyconserving trajectories, calculations tend to converge from below rather than from above experimental energies.

Readers should be reminded that the evolution of the wave function by solving (12) is not an initial-value problem, although it may appear to be from the methodology just outlined. This is a point of confusion for workers whose experience is in time-independent structure theory. In fact the Heisenberg uncertainty principle, $\Delta E \Delta t \geq \hbar$, guarantees that at initial time the spectral width of a line is infinitely broad and narrows with the length of the time integration, whose measure is $\Delta t$. The positions of the spectral peaks (Figure 3 ) are accurately predicted even for early times and wide spectral lines, as measured by $\Delta E$, but the integration should be continued for long enough times that the spectral peaks for different eigenvalues are well separated. This may be hard to accomplish for integrating (12) for heavy particles.

Figure 1 shows quantum trajectories in the $y$ direction for up and down spin states and identical spatial orbitals at initial time. All calculations are performed for protons located at $\pm R / 2$ along the $z$ axis. These are trajectories which belong to the ${ }^{1} \Sigma_{g}$ state. It is easy to see that the two opposite-spin trajectories are correlated in the sense that they keep apart and on opposite sides of the centers of attraction most of the time. Notice the expanding envelops of the amplitudes with increasing time, which indicates the increasing excited and continuum-orbital contribution to the wave functions with increasing time. This behavior is possible because the time-dependent or spectral wave functions which are used to calculate the currents, whence the trajectories are a superposition of the complete set of states of the Hamiltonians, as emphasized in [10].

Figure 3 shows the spectra at an internuclear distance of $R=2.5$ au as calculated from the temporal Fourier transform of the overlaps of the wave functions at time $t$ with the initialtime wave functions, as discussed in [10]. Having located the spectral energies at the positions of the peaks, eigenfunctions (Figures 7 and 8) are calculated from the Fourier transform of the spectral wave function precisely at the spectral peak positions. A window function [10], $w(t)=\left(1-\cos \left(2 \pi t / t_{\max }\right)\right.$, is used in both the eigenvalue and eigenfunction calculations in order to suppress side-lobe numerical artifacts.

Figure 7 shows a $z$-slice at $x=y=0$ of the eigenfunctions for $R=1.4$ au, and Figure 8 similarly shows the eigenfunctions for $R=2.5 \mathrm{au}$. The fixed locations of the protons are clearly depicted in both plots as the twin peaks 


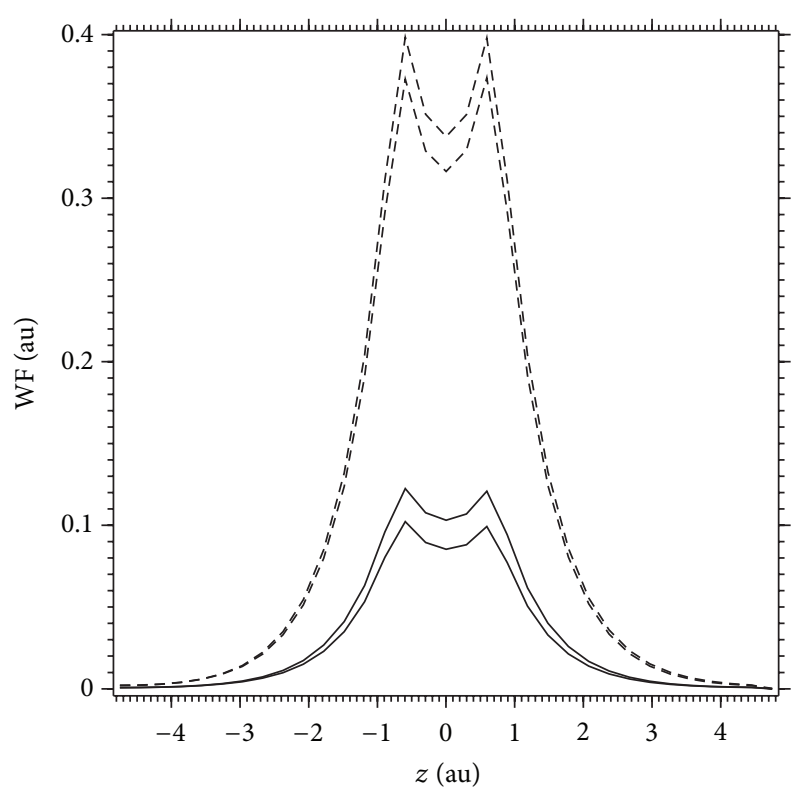

Figure 7: Wave functions along $z$ at $x=y=0$ at $R=1.4$ au for the ${ }^{1} \Sigma_{g}$ state of $\mathrm{H}_{2}$. Solid: spin-up electron. Dotted: spin-down electron. Convergence is shown for the solid curves at $0.75 t_{\max }$ (lower) and for $t_{\max }$ (upper) and for the upper curves at $0.75 t_{\max }$ (upper) and for $t_{\max }$ (lower), where $t_{\max }=200 \mathrm{au}$.

of the eigenfunctions. The nascent separation into $\mathrm{H}$ dissociation products is evident in Figure 8 by the dissymmetry of the wave functions with respect to the nuclear centers. The wave function which is symmetric about the proton centers has the interelectronic potential set equal to zero; thus the correct separation into He-atom dissociation products depends on the correlation of the two electrons. Figure 4 shows corresponding quantum trajectories in the $z$ direction calculated using (7) in the nonrelativistic limit, as in Figure 1, but with the spectral wave function, which is a superposition of states, replaced by eigenfunctions obtained, as mentioned previously and described in [10], from the temporal Fourier transform of the spectral wave function at a spectral eigenvalue. These may be considered to be eigentrajectories, as opposed to the spectral trajectories appearing in Figure 1. Notice that at later times the eigentrajectories for $R=$ 1.4 au are pulled into the internuclear region, reflecting the covalent nature of the bond. In contrast the eigentrajectories for $R=2.5$ au remain in the separated-atoms region for all times, reflecting the weak covalency of the bond for large internuclear separation. We regard this behavior as strong justification for the present methodology, in which the correlation of the two electrons guarantees the correct dissociation products automatically without having to build this behavior into the basis set in a conventional variational calculation.

Figure 2 shows eigentrajectories for electrons having the same spin states and different initial orbitals. These are trajectories belonging to the ${ }^{3} \Sigma_{u}$ state.

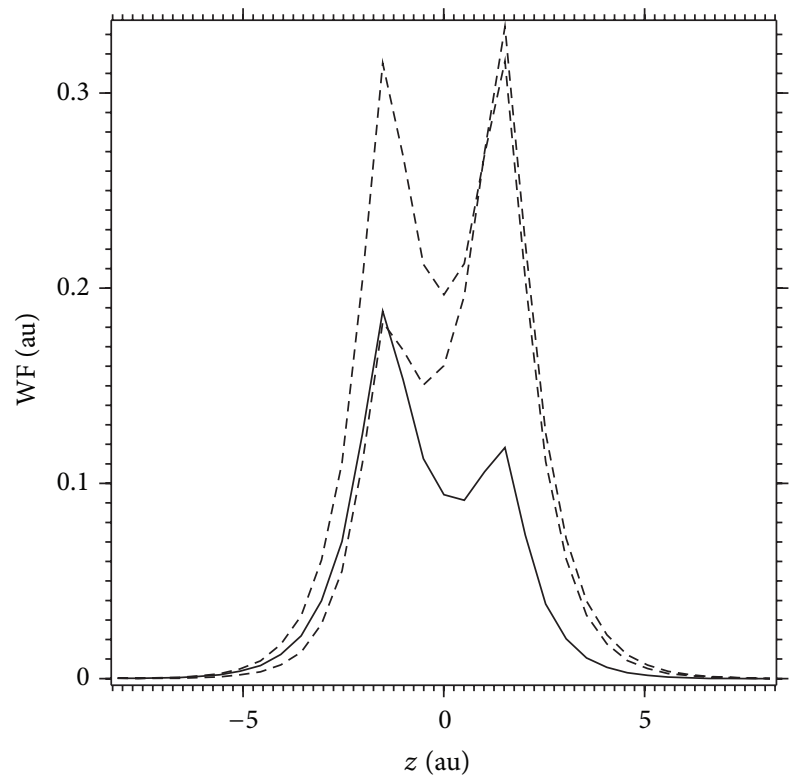

FIGURE 8: Wave functions along $z$ at $x=y=0$ at $R=3.0$ au for the ${ }^{1} \Sigma_{g}$ state of $\mathrm{H}_{2}$. Solid: spin-up electron. Dotted: spin-down electron. The dotted curve which is symmetric about the two proton positions is calculated for the interelectronic interaction artificially set equal to zero, demonstrating that the correct dissociation limit into two hydrogen atoms, as shown by the solid and dotted curves asymmetric about the two protons, depends critically on the interelectronic interaction.

Notice that the trajectories are separated into a groundstate trajectory centered about one nucleus and an excitedstate trajectory moving at large distances from the two centers of attraction. Such a state is known in quantum chemistry as antibonding.

Figure 5 shows the late-time energies of the two states and the time averages of the energies. The energies are calculated from the expectation value of the two-electron Hamiltonian using the eigenfunctions inferred from the spectral solution, as discussed previously and in [10]. The interelectronic potentials are calculated using the eigentrajectories, as discussed previously, and the sum over two electrons is divided by 2 in order to avoid double counting. Notice that the time dependence of the potential, as given by (8)-(9), means that the Hamiltonian expectation values tend to oscillate about a stationary time average, as shown in Figures 5 and 6.

Finally Figure 6 shows the energy time averages for four different internuclear distances. The agreement of the time averages with the variational calculations of Kolos and Wolniewicz [13] is good, whose energies for the bonding and antibonding states are -1.174 and -0.7842 , respectively. Readers are reminded that the binding energy is only $17.45 \%$ of the total electronic energy of -1.174 for the ground state of molecular hydrogen. The agreement at the larger internuclear distances is not as good owing to smaller binding energies whose magnitude falls into the last digit of accuracy of the calculation. 


\section{Conclusions}

One cannot extract more than three significant figures from the calculations at the present level of accuracy, but we are confident that the results can be systematically improved for accuracy by further refinements in the grid meshes and especially by using improved, energy-conserving numerical methods to integrate the quantum trajectories in order to avoid run away, the underestimation of the interelectronic potential energy, and as a consequence the overestimation of the binding energy. These problems notwithstanding, we believe that the quantum-trajectory theory (a) establishes the relationship between the spin of an individual electron and the Fermi-Dirac statistical behavior of an ensemble of electrons and (b) achieves the pair-wise correlation of electrons by evaluating Coulomb's law as an instantaneous interaction in the time rather than as a quantum stationarystate mean or exchange interaction.

\section{Acknowledgments}

The author is grateful to T. Scott Carman for his support of this work. This work was performed under the auspices of the Lawrence Livermore National Security, LLC (LLNS), under Contract no. DE-AC52-07NA273.

\section{References}

[1] H. M. James and A. S. Coolidge, "The ground state of the hydrogen molecule," The Journal of Chemical Physics, vol. 1, no. 12, pp. 825-835, 1933.

[2] M. E. Riley, J. M. Schulman, and J. I. Musher, "Nonsymmetric perturbation calculations of excited states of helium," Physical Review A, vol. 5, no. 5, pp. 2255-2259, 1972.

[3] R. J. Bartlett and M. Musial, "Coupled-cluster theory in quantum chemistry," Reviews of Modern Physics, vol. 79, no. 1, pp. 291-352, 2007.

[4] D. Bohm, Physical Review A, vol. 85, p. 166, 1952.

[5] P. Holland, Quantum Theory of Motion, Cambridge Press, Cambridge, UK, 1993.

[6] R. E. Wyatt, Quantum Dynamics with TrajecTories: Introduction to Quantum Hydrodynamics, Springer, New York, NY, USA, 2005.

[7] J. D. Bjorken and S. D. Drell, Relativistic Quantum Mechanics, McGraw-Hill, New York, NY, USA, 1964.

[8] H. A. Bethe and E. E. Salpeter, QuantumMechanics of One- and Two-Electron Atoms, Dover, New York, NY, USA, 2008.

[9] H. A. Bethe and E. E. Salpeter, Quantum Mechanics of One- and Two-Electron Atoms, Dover, New York, NY, USA, 2008.

[10] M. D. Feit, J. A. Fleck, and A. Steiger, "Solution of the Schrödinger equation by a spectral method," Journal of Computational Physics, vol. 47, no. 3, pp. 412-433, 1982.

[11] M. E. Riley and B. Ritchie, "Numerical time-dependent Schrödinger description of charge-exchange collisions," Physical Review A, vol. 59, no. 5, pp. 3544-3547, 1999.

[12] B. Ritchie, "Quantum molecular dynamics," International Journal of Quantum Chemistry, vol. 111, no. 1, pp. 1-7, 2011.

[13] W. Kolos and L. Wolniewicz, "Potential-energy curves for the $X^{1} \Sigma_{g}, b^{3} \Sigma_{u}$, and $C^{1} \Pi_{u}$ states of the hydrogen molecule," The Journal of Chemical Physics, vol. 43, no. 7, pp. 2429-2441, 1965. 

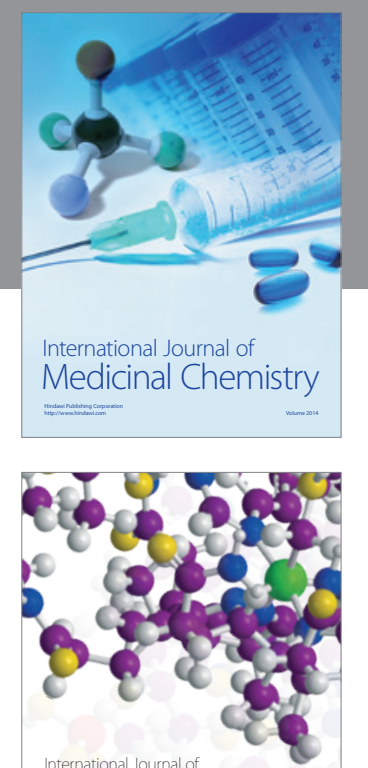

\section{Carbohydrate} Chemistry

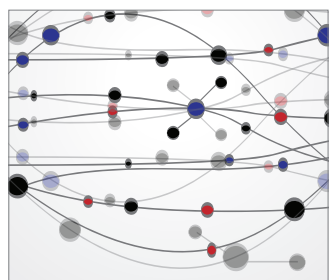

The Scientific World Journal
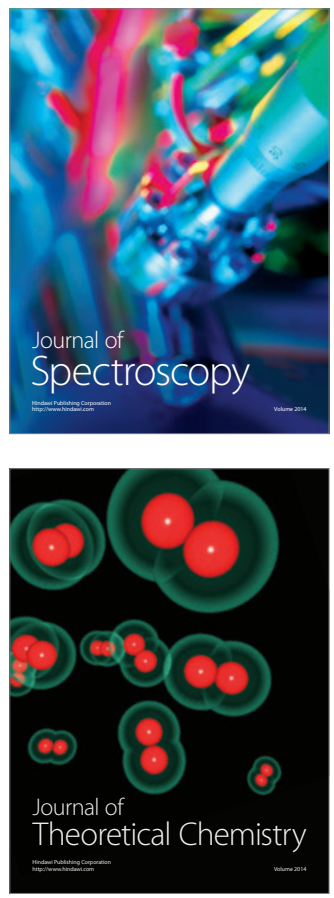
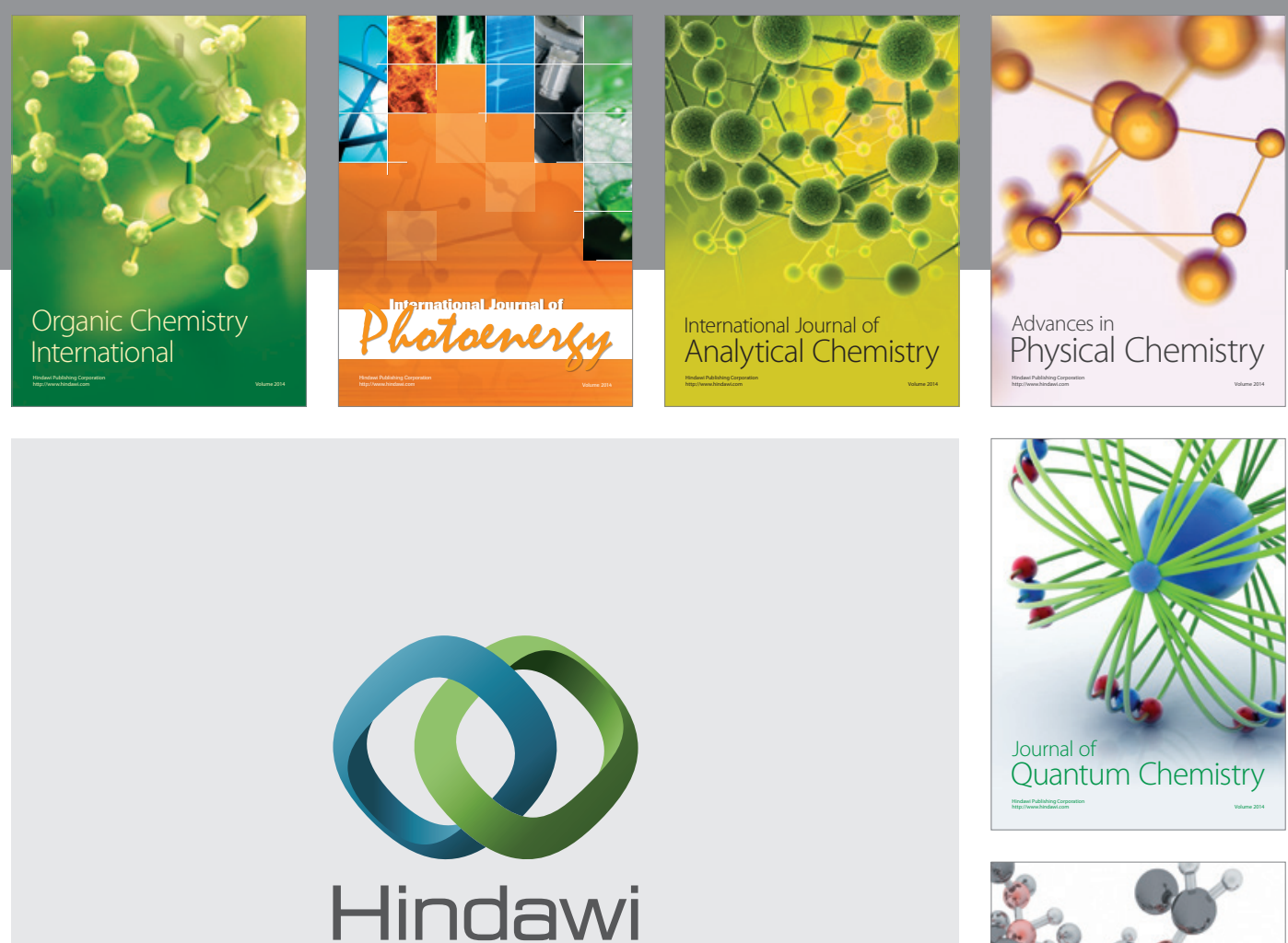

Submit your manuscripts at

http://www.hindawi.com

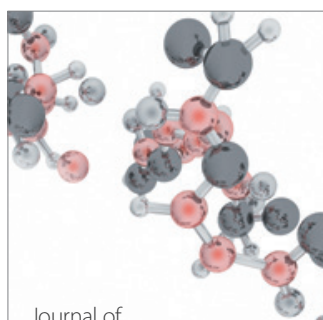

Analytical Methods

in Chemistry

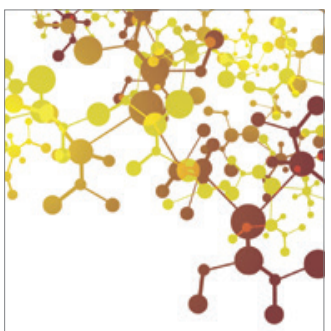

Journal of

Applied Chemistry

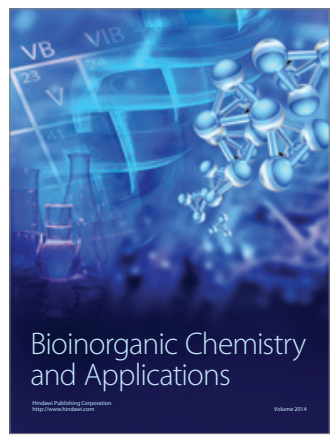

Inorganic Chemistry
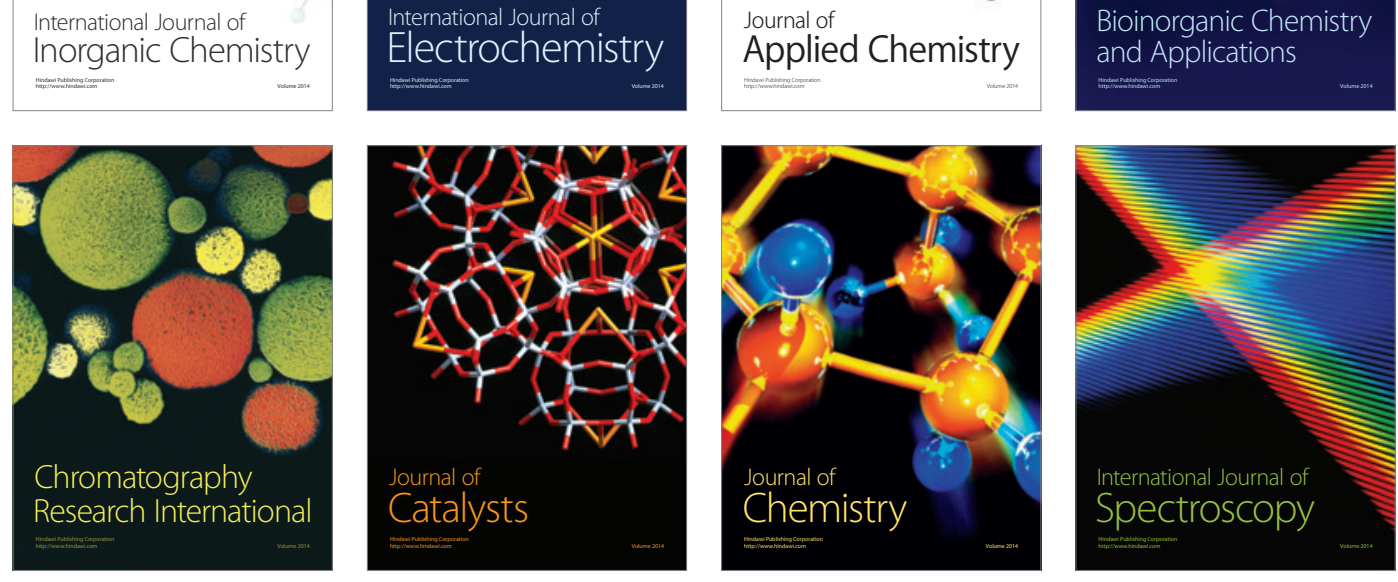